\title{
Quality Assurance of Picture Archiving Communication Systems With Laser Film Digitizers
}

\author{
Peter D. Esser, Ethan J. Halpern, and E.S. Amis, Jr
}

\begin{abstract}
A comprehensive quality assurance (QA) program should be implemented for all teleradiology and picture archival and communications (PACS) systems. In this report, we summarize our $Q A$ experience with a teleradiology system that includes a laser digitizer for $x$-ray film. A key component required for the evaluation of laser film scanners is an appropriate test pattern; digitizers should be evaluated with enhanced test patterns specifically designed for this purpose. The phantom pattern should measure high-contrast resolution, low-contrast discrimination, gray scale linearity, geometric distortion, and noise. In addition, a uniformly exposed sheet of film lapproximately 0.3 optical density) serves as a good phantom for testing screen nonuniformities of viewing station monitors. It is also suggested that clinical images should be included in a OA program. Finally, it is recommended that any discrepancies in the interpretation of teleradiology images should be monitored and investigated.

Copyright 1991 by Society of Photo-Optical Instrumentation Engineers
\end{abstract}

\begin{abstract}
A DAILY quality assurance (QA) program should be part of the operation procedures of all picture archival and communications (PACS) systems. In a system with a laser film digitizer, the physician at the viewing terminal is placing his clinical trust in the electronic representation of an $x$-ray image that has been digitized by a complex electromechanical device, processed by the device's dedicated computer, compressed, transmitted, archived, decompressed, and, lastly, displayed at the highresolution physician's viewing station. Many variables exist in the complex imaging chain, such as the size and shape of the laser beam, calibration of the photon detection system and the presence of any dust on the optics. The viewing monitor itself must be properly aligned, with a uniform phosphor, and brightness and contrast must be adjusted correctly. Thus, loss
\end{abstract}

From the Center for Computer Applications in Radiology, Columbia Presbyterian Medical Center, New York, NY.

Presented at Medical Imaging V, San Jose, CA, 1991.

Address reprint requests to Peter D. Esser, Center for Computer Applications in Radiology, Columbia Presbyterian Medical Center, New York, NY.

Copyright $\odot 1991$ by Society of Photo-optical Instrumentation Engineers.

0897-1889/91/0404-0007\$03.00/0 of information and deterioration of image quality can occur at numerous places in a teleradiology system. To assure optimized performance, a quality assurance program should test all components of the system-from the digitizer to the display station. Unfortunately, however, at this time there is no generally accepted QA protocol for teleradiology or PACS systems.

Previous articles have evaluated the performance of laser digitizers. ${ }^{1-4}$ It also has been demonstrated that the complex mechanical, optical, and electronic components in a highresolution laser digitizer can introduce a number of unusual artifacts into a digital image; for example, a streaking artifact from bright areas into adjacent dark regions. ${ }^{5}$ To aid in the identification of these artifacts and to provide a suitable phantom for QA, an improved phantom for laser scanners has been developed by Halpern and Esser. ${ }^{6}$ In addition, the Society of Motion Picture and Television Engineers (SMPTE) ${ }^{7}$ pattern is often used as a QA phantom for PACS. In this article, we summarize our practical experience in implementing a routine, daily quality control program for a teleradiology system that includes a physician's viewing station and a laser film digitizer.

\section{EQUIPMENT}

The teleradiology system installed at the Columbia Presbyterian Medical Center consists of a CommView digital system (AT\&T Bell Laboratories, West Long Branch, NJ and Philips Medical Systems, Shelton, CT) with a laser digitizer (model FD2000, Dupont, Wilmington, DE) located at a satellite hospital 4 miles from the medical center. A dedicated T-1 line links the systems. The primary purpose of the system is to provide evening and weekend radiology coverage for the remote location. At a separate location in the medical center, a data management computer system provides long-term archival storage and maintains the patient database. The physician's viewing station consists of an independent computer system for local image storage and a high-resolution display system with two monitors $(1,024 \times 842 \times 12)$; full reso- 
lution images $(2,048 \times 1,684$ pixels $)$ are displayed by magnifying portions of the image. An important feature of the system is a separate Ethernet port, which can be used to down-load images for numerical analysis at workstations (an enhanced IBM AT and a Macintosh FX) in the Radiology Department.

\section{QUALITY ASSURANCE}

The primary phantom for the laser digitizer is described in detail in a companion article. ${ }^{6}$ In summary, it consists of a 4,984 × 4,084-pixel matrix with various patterns to measure resolution, low-contrast discrimination, gray scale response, and geometric distortion. The test pattern is printed digitally on a $14 \times 17$-inch sheet of film with a $1 / 2$-inch black margin. For routine daily testing a copy of the original pattern is used. In addition to this phantom, a sheet of partially exposed $\mathrm{x}$-ray film (approximately 0.30 optical density) is also digitized. Sample clinical images are routinely used. Occasionally, the SMPTE pattern is used as well.

Quality assurance should be performed daily. We have found it advantageous to transmit and display three or more images. This provides images for both monitors and tests the system's archival and retrieval capability. One image is always an enhanced pattern image described previously and the second is a partially exposed sheet of film. The latter is particularly useful in the daily evaluation of the monitor screen uniformity. In addition, this also tests for the presence of low-frequency patterns originating from the digitizer. The black border surrounding the Halpern phantom is important in detecting light leaks. The primary method of evaluation is by visual inspection. However, we are planning to supplement this by periodic numerical analysis of parameters such as gray scale values and image noise. It should be noted that the ability to quantitate phantom values was of major importance in acceptance testing. We also have found that most physicians prefer viewing several clinical images as an adjunct to the test patterns. Finally, it is recommended that the results of the daily quality assurance be recorded in a log book.

Most electronic problems such as board failure generate catastrophic failures of the PACS system that are relatively easy to identify. How- ever, other malfunctions, such as light leaks, small changes in the laser beam, and dust on the optical components of the digitizer, require careful inspection of the phantom image. Many of these problems can be reduced by a preventive maintenance program. In addition, there can be operational problems associated with the use of the laser scanner. These include dirty films (especially handprints), bent films, and incorrect orientation. Whereas these may be rare, the viewer must be aware that they can occur occasionally.

Based on our experience, it is recommended that an orientation and training program be available for all new physician and technologist users of the system. In addition, it is advantageous to have a system consultant available.

Quality control of the teleradiology hardware should also be supplemented by a clinical QA (or as it is currently coming to be known, quality improvement) program. This is probably best done using the 10-step program required by the Joint Commission on Accreditation of Healthcare Organizations (JCAHO). The clinical indicator in such a program would be the capability of the transmitted image to accurately demonstrate all findings present and discernible on the original hard copy. Monitoring of this indicator can be accomplished by comparison of independent interpretations of both the original hard copy study and the transmitted images. The threshold should probably be $0 \%$, because persistent misinterpretations based on the transmitted image degrades the quality of patient care and mandates improving the teleradiology system. In other words, all discrepancies should be reviewed to determine if there are specific types of studies in which the accuracy declines, such as an inability to detect subtle pneumothoraces on chest radiographs. If such a problem area were to be consistently identified, it could be concluded that such studies should not be transmitted for interpretation.

In our institution, each transmitted case receives a preliminary interpretation at the medical center. This result is then available to the radiologist reading the original study the next morning. If a discrepancy is noted, the referring physician (usually in the emergency room) is notified and the patient recalled if necessary. A record is kept of these cases. Of approximately 
3,000 cases transmitted during the first 2 years of operation of our system, serious discrepancies in interpretations of the transmitted hard copy studies affecting patient care were identified in only nine instances. The nine cases represented a mix of typical emergency room studies.

To test the system, the nine discrepant cases were mixed with 10 other randomly selected studies and the transmitted (digitized) images viewed independently by three board-certified radiologists. Each radiologist was allowed to manipulate the digitized image as desired before recording a diagnosis. In all nine cases in question, enough information was available on the image to reach the correct diagnosis. However, it must be noted that image manipulation, predominantly magnification and contrast reversal, were useful in identifying subtle findings such as hairline fractures. Distribution of the results of this study showing excellent correlation between digitized and hard copy images was useful in increasing confidence in the system on the part of residents and staff alike.

Rather than a retrospective review of clinical accuracy of a teleradiology system, when a new system is being installed it would be wise to transmit a statistically significant number of various types of cases and pathologies and then compare the blinded interpretations of the digitized images with those of the originals. This would provide documentation at the time of startup of a system that it is functioning in a clinically satisfactory manner.

\section{ACKNOWLEDGMENT}

We thank AT\&T for their assistance with the Ethernet link to the CommView system, and George Knox for his help in supporting the quality assurance program and systems operation.

\section{REFERENCES}

1. Takeuchi H, Taira RK, Huang HK, et al: Preliminary experiences with a laser scanner and printer system for radiological images. SPIE Proc PACS III 539:65-71, 1985

2. Lo SC, Taira RK, Mankovich NJ, et al: Performance characteristics of a laser scanner and laser printer system for radiological imaging. Comput Radiol 10:227-237, 1986

3. Vranck J, Strul B: Performance evaluation of a laser digitizer. SPIE Proc Med Imaging 767:524-528, 1987

4. Lo SB, Gakill JW, Mun SK, et al: Contrast information of digital imaging in laser film digitizer and display monitor. J Digital Imaging 3:119-123, 1990

5. Halpern EJ, Esser PD, Nickoloff E, et al: Artifacts introduced by laser scanner digitization of radiographs in Arenson RL, Fredenberg RM (eds): SCAR 90. Computer Applications to Assist Radiology (Proceedings of the 10th Conference on Computer Applications in Radiology). Anaheim, CA, Symposia Foundation, 1990, pp 276-284

6. Halpern EJ, Esser PD: An improved phantom for quality control of laser scanner digitizers in PACS. Medical Imaging V: Image Capture, Formatting, and Display. SPIE Proc Med Imaging 1444:104-115, 1991

7. Gray JE, Liks KG, Haddick DH, et al: Test pattern for video displays and hard-copy cameras. Radiology 154:519527,1985 